\title{
A Demand for Data to Improve Outcomes Creates the Why to Move to Third Generation Immunization Information Systems
}

\author{
Todd Watkins ${ }^{1 *}$, President, Bachelors Systems and Industrial Engineering (BSIE) \\ Michael Popovich¹, CEO, Masters of Science Systems Engineering (MSSE)
}

Kristina Crane ${ }^{1}$, Director of Product Strategy and Project Management Office, Bachelor of Arts (BA)

${ }^{1}$ Scientific Technologies Corporation, 411 S. $1^{\text {st }}$ Phoenix, Arizona 85004

\begin{abstract}
Investments over the past two decades to collect and store immunization events established a national population health data asset. The ability to track vaccine usage and storage has increased accountability, lowered wastage, protected valuable resources, and provided the correct vaccines at the right time.

Sixty-four immunization registries support the current immunization ecosystem, yet all investments to date have been through state and federal funding. Much of the technology supporting these registries is becoming harder to support, limiting the utilization of the data. For the most part all current systems have legacy 2 nd-generation technology and architectures as their foundation.

Current technology investments in these national assets tend to be for systems that within the next five years will not be cost effectively sustainable with only federal, state and local funding. Yet quality data is being reported by immunization providers across the health care network that is increasing exponentially through electronic data exchanges integrated within Electronic Health Records (EHR) and Pharmacy Management Systems (PMS).
\end{abstract}

This increase in high-quality patient immunization records creates opportunity to build immunization intelligence from the data. However, 2nd-generation Immunization Information Systems (IIS) limit the effective and timely use of this information. Considering the increasing value of the data to public and private sectors working to close immunization care gaps in populations, supporting technology must ensure easy access.

This is the first of two papers that highlights the power of these national registries and the data they contain to provide opportunity intelligence to the immunization ecosystem user community. Paper one illustrates the "why" for change and the need for a truly community collaborative path forward to move from 2 nd- to 3 rd-generation systems through partners that leverages cost sharing and common goals.

The end goal is to establish new supporting technology assets that accelerate the use of data to impact vaccine preventable disease (VPD) outcomes which create a new model for public-private investments to sustain the IIS national infrastructure. The second a working paper with assumptions to be tested 
("A Model for Sustaining and Investing in Immunization Information Systems"), shares cost and investment strategies to complete the migration and create sustainable immunization systems for the future.

*Corresponding author: Todd watkins@stchome.com

DOI: 10.5210/ojphi.v11i2.9412

Copyright @2019 the author(s)

This is an Open Access article. Authors own copyright of their articles appearing in the Online Journal of Public Health Informatics. Readers may copy articles without permission of the copyright owner(s), as long as the author and OJPHI are acknowledged in the copy and the copy is used for educational, not-for-profit purposes.

\section{Introduction}

"We choose to go to the moon. We choose to go to the moon in this decade and do the other things, not because they are easy, but because they are hard, because that goal will serve to organize and measure the best of our energies and skills, because that challenge is one that we are willing to accept, one we are unwilling to postpone, and one which we intend to win, and the others, too." [1]

In 1962, President Kennedy established a national goal. America's Neil Armstrong and Buzz Aldrin landed the lunar module Eagle on the moon on July 20, 1969. Six hours later, Armstrong took his famous step, followed less than thirty minutes later by Aldrin [2]. In just seven years the mission had been accomplished; with a first generation lunar lander.

In 1990, the Centers for Disease Control established a national goal to create childhood immunization tracking systems [3]. These data systems would allow pediatricians and family physicians to record in a state-based central repository the immunization histories of their patients; allowing any physician to know what a child needed on future visits. Within five years this goal had been achieved. First generation childhood immunization tracking systems were being implemented in state public health departments [2].

Both the manned space missions and the changes in childhood tracking systems have evolved as technology and missions changed. The U.S. has focused on space shuttle orbital missions. Registries for kids have become Immunization Information Systems (IIS) for all. Technology and applications have been required to keep pace in space and continue to deliver high quality solutions health information to the immunization community.

The U.S. today continues to invest in technology and people to diminish the impact of vaccinepreventable disease (VPD) on populations - a public mission equally as challenging as going to into space, the moon and soon beyond. Immunization technology (IT) systems today are required to support increasing numbers of users. With over two decades of data contained therein they are 
poised to deliver immunization intelligence to ensure population health vaccine programs produce positive outcomes.

However, the use of data and system expansion is challenged by existing IIS architecture legacy designs and technology environments supporting current systems. The increasing costs to maintain, evolve to meet new vaccine recommendations, increase vaccine utilization accountability, diseases and access data in a timely fashion are leading indicators to suggest a next generation of technology is required.

\section{Historical Context}

There are sixty-four state, local and territorial IIS. Each is independent. Each supports the immunization programs and community of users within their jurisdictions. Many that began in the mid-90s used first generation designs and tools to build and implement. The technology was based on client server technology, local area networks and dial-up telecommunications capturing data content focused exclusively on childhood immunization events.

As the internet advanced and became mainstream, 2nd-generation technical IIS solutions evolved. Public health IIS vendors and state IT staff moved into the dot com world for connectivity and online data input. This was generation 2 versus desktop applications in provider offices that upload immunization events in batch operations (generation 1).

Over two decades the nation's IIS have grown in the data that is being collected and in new features to support changing vaccine schedules, reporting requirements and expanding user communities from traditional immunization providers to pharmacists, school nurses, and first responders. From 1st to 2nd generation technology solutions, the software changes required to support these changes continued to be built on a single code base.

Today, these systems include features to support vaccine inventory management and ordering, electronic data exchange with EMRs and pharmacy systems, patient reminder/recall, identification process and complex de-duplication algorithms to match patients and their immunization events. These systems include components for the Vaccine for Children (VFC) provider enrollment efforts, school nurse and day care tools that allow review of student vaccination records. Current ACIP guidelines providing clinical decision support and enhanced security to protect data are common improvements made though out the year.

Each state has invested and grown their IIS code base to support these features. Today it makes ongoing sustainability and data quality an increasing challenge. In short, these systems and their supporting infrastructures are getting old. The public health IIS is a cornerstone of population health data and is a significant information asset. The key is the ability to effectively utilize the data. Existing 2nd-generation IIS architectures, software, and processing technology has become a barrier to this goal.

Consider the technology environment we all live in today. We have seen the rapid uptake of smartphone technology and the increasing use of voice interaction. Cloud environments supported 
by private technology companies are the new normal for hardware servers. The push for electronic health records and sharing with patients is driving the private sector investments and the demand for ease-of-use, integration of predictive modeling, machine-learning, use of data for decision making, and artificial intelligence in the health care field is accelerating the need for better data.

Software engineers and developers have watched an even more rapid evolution of programming languages, database architectures, development tools, and engineering protocols. Today's lexicon words such as agile, microservices, serverless architecture, DevOps, blockchain, internet of things, quantum computing, on-demand APIs, actionable analytics, pair programming, ecosystem, collaboration, social media, and full stack development are common. However, these words do not describe today's technology and the human resources supporting public health IISs, the data contained therein, and the users connected across their networks.

Comments such as, "Our technology is too old"; "The cost to sustain the IIS technology is growing faster than budgets allow"; "The quality, reliability, and performance are diminishing"; and "We don't have the staff needed to use the data to impact behavior" are frequently heard. Yet when discussions move toward change, the most common comment heard is, "We are fine as is - our users are happy the way it works now."

When President Kennedy put forth his moon mission in 1962, few believed it was possible. It was not uncommon to hear "We are fine the way our space program works now." But it was the time for leadership. Kennedy put forth and invested in a unifying mission.

\section{Legacy-Free by 2023: The Challenge}

Changing from legacy systems that have been operational for years to $3^{\text {rd }}$ generation registries will not be economically feasible without a collaborative and common approach. The unifying mission for this change is to recognize that the intelligence derived from the data in IIS is the key to influence. Influence clinical providers and pharmacists to close patient immunization care gaps. Influence individuals to close their vaccine gaps, complete their vaccine series or get their annual flu shots. A proactive mission based upon data in the IIS that improves population outcomes will demonstrate investment value and maintain future sustainability.

With this as a unifying mission, in June 2017, a collaborative of twelve state immunization program leaders met to address current existing IIS challenges. As they shared their stories it became clear that their immunization registries were not cost effective to maintain with the present technology and budgets. In all cases what started as a single commercial-off-the-shelf (COTS) solution evolved into 12 custom environments. Even though all were built on the same code base, each state program implemented a different release of the system. Every IIS looked the same, but each ran very differently. There was no consistency for the 150,000 users of these systems.

A persistent problem was some states took more than a year to implement the current release of their COTS system into production. As a result, product updates and bugs that had long been fixed in the software were still causing issues for the states that lagged behind. State IT environments that hosted their own IISs varied greatly from one state to the next and had policies that often 
resulted in delayed roll-outs of releases or patches. Users continued to report the same errors in one state that were no longer an issue in another state. While provider end users were unhappy at the pace of updates to the IIS, the health departments' immunization programs were overwhelmed with the frequency of application releases. Immunization registry managers struggled with finding resources to continuously perform user acceptance testing to move a release from their testing environment into production. Documentation teams could not keep up with updates.

The IIS customer support teams required a unique knowledge base for each individual client. The bottom line: To address the immediate complaints, individual concessions had to be made for each state based on the version of the COTS system they were on, the way each IIS database was uniquely structured, and the IT environment upon which the IIS was hosted.

This June 2017 meeting marked the first time twelve state participants decided to start working toward a new approach [4]. The vision was to create a single release of an IIS to be used by all an IIS that would leverage the cloud for hardware and performance, a redesigned architecture that would allow for data optimization, and rapid updates of software. The cloud-hosted IIS would be one common version supported by agile teams that include public health leadership. The framework took best practices from the technology industry, including clear partnerships based on the SaaS business model. This would provide added clarity and accountability to both the technical support teams and the public health departments receiving the service.

The challenge created by the collaborative also included the requirement for a common modernized system that improved workflows, data capture and quality, tools to enhance user interaction and patient outreach. The IIS functionality challenge was to also take advantage of rapid technology advancements and IT tools and platforms that allow for employment of modern software engineering principles, notably as automated testing, continuous deployment (DevOps), microservices, web services and APIs - each of which are developed, revealed, and integrated simultaneously with each code update. The core of this foundation should seamlessly enable interoperability and data sharing, providing powerful incentives for investment by private sector stakeholders.

This challenge is illustrated in Figure 1. Convert integrated "monolithic" immunization systems of today to an architecture and set of functions assessable by all through shared services. And to achieve this legacy conversion by 2023. 

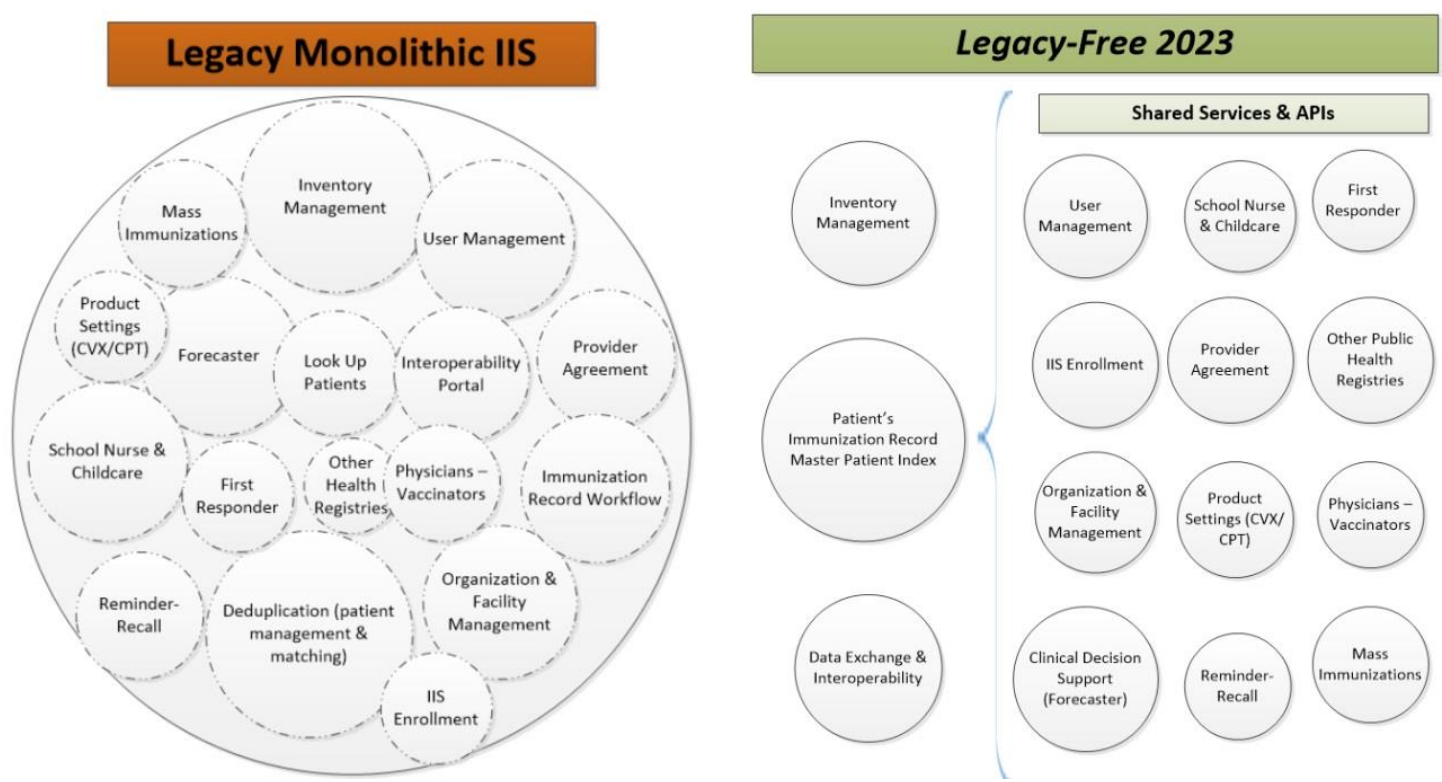

Figure 1 - The Technical Challenge to Move from $2^{\text {nd }}$ to $3^{\text {rd }}$ Generation Immunization Information Systems

The value of this new design approach can be illustrated by an example: decision support which is required by all 64 custom systems today to determine immunization care gaps for individuals. On-going ACIP updates require states to implement update forecasting tools in their systems. These updates once implemented are now integrated into each individual system. With a Web Services model an update to a single version of a forecasting tool would be deployed to every $3^{\text {rd }}$ generation IIS in minutes without affecting other aspects of the IIS.

Compare that speed and efficiency to today's model, where a traditional Software Change Request is written, an estimate is given, the forecaster code that is buried somewhere in the IIS is modified, the entire IIS is tested to make sure the changes do not negatively impact another part of the monolithic IIS, and then a patch is applied to the IIS and the product is released.

In a recent Forbes business article, "technology changes at a rapid rate due to competitive innovation, lowered implementation costs, and facilitator technologies - such as the cloud and mobile devices... To integrate new technology at a reasonable pace, many organizations can no longer afford to coddle or work around late majority and laggard thinking, and one visionary leader isn't enough to push a company into a culture of change. Individuals throughout the organization need to demonstrate the innovator, early adopter, and early majority traits in order to execute a leader's vision." [5]

As noted by Forbes, lower implementation costs also extend to lower support costs. The 2nd generation of IISs can no longer be expected to grow and serve the hundreds of thousands of users - who access this information daily - with the current level of funding. They no longer can be expected to support accelerated electronic data exchange with clinical providers, pharmacists, and 
public health clinics to the extent that they are able to mitigate the impact of an outbreak or the next pandemic.

\section{The Role of the Consortium}

The distinction between first to 2nd IIS evolution and now the 3rd is the process that was used and what is now required to achieve success. Technology drove the first phase of evolution. Vendors and government IT professionals simply continued to adhere to the registry use cases (an old school approach) and standards while taking advantage of a new interconnected communication environment through the internet. In spite of the dramatic reach the internet provided from the first to 2nd generations, registries were still about data collection and storage. Product owners were non-existent, and public health did not participate as a community to fully leverage the power the internet and all its emerging technologies provided for the purposes of growing the IIS beyond its data gathering roots.

Today that paradigm has shifted. The drivers of the transition today are (1) demands for data to deliver immunization intelligence across the ecosystem; data used to measure population health outcomes, engaging the provider community and empowering individuals; (2) managing the cost of these systems and creating an environment to encourage private and public investment; and (3) ensuring systems are secure and information is protected through software and framework updates to reduce and close access gaps to the unauthorized.

First to 2nd generation migration efforts were typically led by the IT organization of state and under the oversight of Chief Information Officers (CIOs). Due to the fundamental shift from gathering data to disseminating the immunization intelligence required of the modernized IIS, this move from the 2 nd to 3 rd generation need to be led by a new type of $\mathrm{CiO}$ - the Chief immunization Officer.

The 12-state consortium that established the unified mission in 2017 has now assumed community accountability for moving forward creating this new IIS framework. The new mission required a common mind shift. It is no longer about the technology but the outcomes and how to achieve real goals long sought after when the vision for the first childhood registry was established. The legacy and the new approach transitioning to an IIS consortium support model as illustrated in figure 2. 


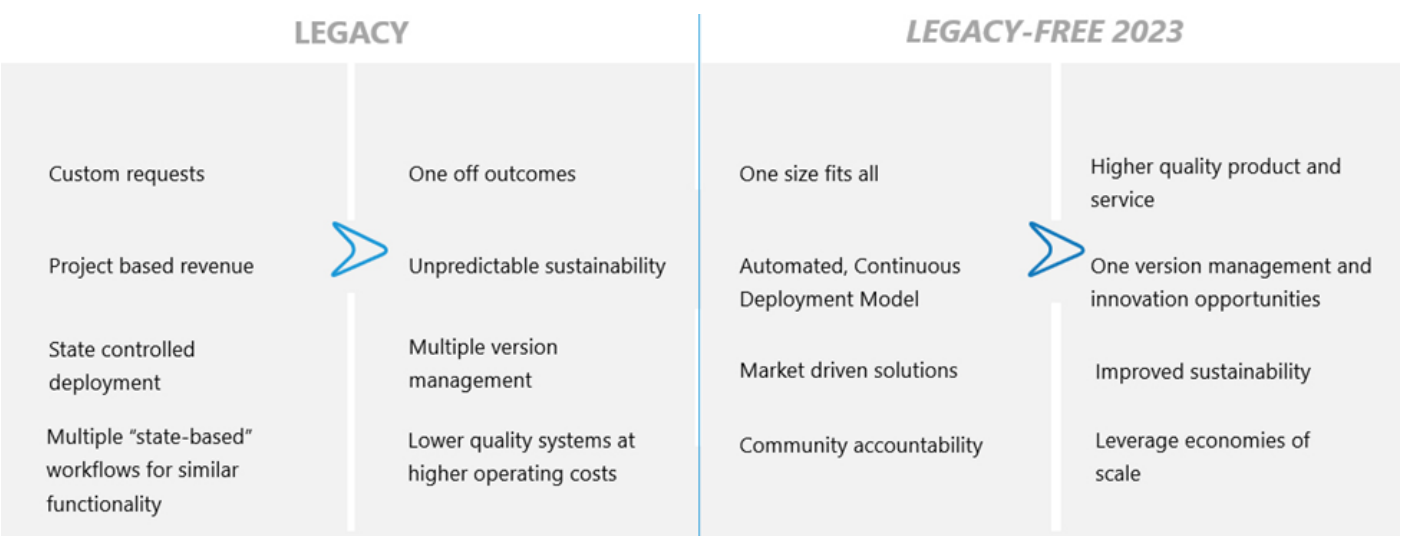

Figure 2: Creating a $3^{\text {rd }}$ Generation IIS Requires a Community and Engaged Consortium Approach

Step one in the process has been for the Consortium to support the move from traditional state government "on premise" servers to private, cloud-based hosting services. This creates the opportunity for DevOps team to deliver updates faster across each system and in real time add capacity to ensure performance under any user demand. This is only step one. A complete modernization of the architecture and software tools for the IIS is required. The role of the collaborative as a shared commitment is to oversee modernizing the user experience, improved work flows and set priorities for feature function removal, updates and delivery.

The technical team supporting the collaborative assumes the role of re-architecting the design, integration points and database to decouple software code into the required feature functions and sharing the progress with the collaborative as each update becomes available to each IIS. Transparency by both the collaborative program and technical teams to communicate with the entire IIS community and immunization ecosystem is also important to share in lessons learned, successes, risks and agile delivery techniques if after the first 12 have implemented a $3^{\text {rd }}$ generation platform the remaining 52 wish to move in this direction.

A key role of this process is as a community to begin to socialize the concept of cost sharing, demonstrating the immense value of the data in the IIS, the ability for rapid access and utilization to support day-to-day coverage challenges, current outbreaks or a pending pandemic. This is taking the message to the public - private immunization community that a $3^{\text {rd }}$ generation IIS is capable of supporting your individual immunization care gap goals and which in turn is the beginning of discussions to establish an approach to potential new public - private partnerships that include investment contributions.

\section{Limitations}

The overall hypothesis is there now is a sense of urgency that in order to sustain the investment that has been made in public health immunization information systems that truly do capture birth to death immunization events from all populations, we have reached a cross roads where the evolution to new technologies is essential. The hypothesis assumes change is necessary to improve the user experience, access to data, security and future integration. It assumes when these new 
platforms are established and implemented that other than the government will be open to discussions to support their sustainment. It also assumes the government is open to these discussions. However, who pays is 2ndary. The first question is will these health data assets 2nd to none in the U.S. be retained.

The only limitation is the will of the community to move forward together versus the traditional silo approaches in the past. This paper suggested only the "why." A 2nd paper illustrates the total cost to move 64 immunizations systems to $3^{\text {rd }}$ generation products while maintaining existing systems.

\section{Summary}

The key requirement to stimulate this change is that public health registries must maintain high quality, secure, and reliable data complete with real-time bi-directional updates as immunization events occur. They must support interoperability and data sharing consistent with HIPAA rules and regulations. They must be the trusted source of clinical decision support at the point of care for all immunizing providers in order to close the gap on due / past-due vaccines. They must also support engagement and empowerment through the appropriate dissemination of immunization intelligence to all stakeholders most importantly individuals.

There is little doubt that this 3rd-generation IIS platform will be the catalyst for achieving full data utilization, resulting in improved immunization coverage across all ages. This platform will provide easy and ready access to crucial encounter-level and aggregate information in support of each user's role in the ecosystem, thereby reducing the impact of VPDs.

A strong partnership is required with all members accountable for the success of the whole. New technology will certainly prove to be a significant asset for the next decade or more, but it will be the power of the data in these systems that will have the lasting impact the CDC intended when they established a national mission for IIS in the early 1990s.

\section{References}

1. Woodfill J. 2012. John F. Kennedy Moon Speech - Rice Stadium. Available from https://er.jsc.nasa.gov/seh/ricetalk.htm.

2. Bogo J. 2018. The Oral History of the Apollo 11 Moon Landing. Available from https://www.popularmechanics.com/space/moon-mars/a4248/oral-history-apollo-11/.

3. Centers for Disease Control and Prevention. 2012. Immunization Information Systems (IIS). Available from https://www.cdc.gov/vaccines/programs/iis/about.html.

4. STC Annual User Group Meeting, Phoenix Arizona, June 2018. Collaborative working sessions to explore the IIS go-forward plan.

5. Newman D. 2016. Why You Should Align Your Business Transformation To The Adoption Bell Curve. Forbes. Available from 
https://www.forbes.com/sites/danielnewman/2016/05/31/why-you-should-align-yourbusiness-transformation-to-the-adoption-bell-curve/\#2fc4aa721160. ${ }^{2}$ One example is the state of Arizona who first piloted a childhood tracking system in 1993. This Arizona Statewide Immunization Information Systems (ASIIS) was operational statewide in 1995. 